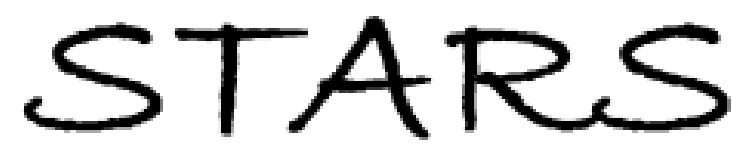

University of Central Florida

STARS

$1-1-1997$

\title{
A renormalization group for dynamical triangulations in arbitrary dimensions
}

Ray L. Renken

University of Central Florida

Find similar works at: https://stars.library.ucf.edu/facultybib1990

University of Central Florida Libraries http://library.ucf.edu

This Article is brought to you for free and open access by the Faculty Bibliography at STARS. It has been accepted for inclusion in Faculty Bibliography 1990s by an authorized administrator of STARS. For more information, please contact STARS@ucf.edu.

\section{Recommended Citation}

Renken, Ray L., "A renormalization group for dynamical triangulations in arbitrary dimensions" (1997). Faculty Bibliography 1990s. 2072.

https://stars.library.ucf.edu/facultybib1990/2072

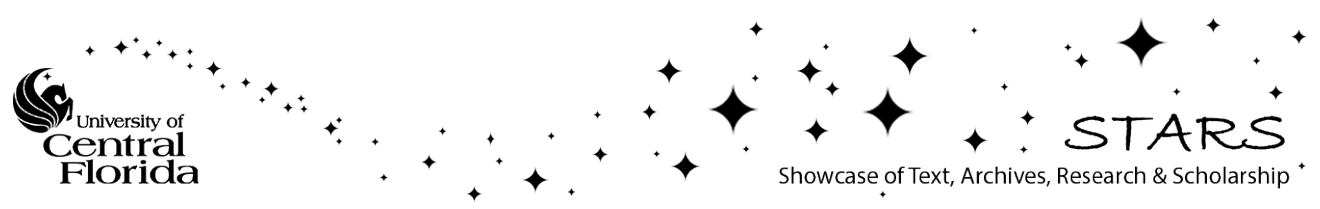




\title{
A renormalization group for dynamical triangulations in arbitrary dimensions
}

\author{
Ray L. Renken \\ Department of Physics, University of Central Florida, Orlando, FL 32816, USA
}

Received 30 July 1996; accepted 29 October 1996

\begin{abstract}
A block spin renormalization group approach is proposed for the dynamical triangulation formulation of quantum gravity in arbitrary dimensions. Renormalization group flow diagrams are presented for the three-dimensional and four-dimensional theories near their respective transitions.
\end{abstract}

PACS: $04.60 .+\mathrm{n} ; 05.70 \mathrm{Jk} ; 11.10 . \mathrm{Gh}$

Keywords: Renormalization group; Dynamical triangulations; Quantum gravity; Critical phenomena

\section{Introduction}

There are currently many different avenues to a theory of quantum gravity. This paper focuses on the formulation provided by dynamical triangulations. Extensive work has been done in two dimensions and a significant body of work has also examined three and four dimensions [1]. Dynamical triangulations are similar to the formulation of Regge in that the manifold is broken up into simplexes and the curvature is identified with a deficit angle about a subsimplex with dimensionality two less than that of the manifold. The formulations differ in their treatment of the functional integral over all possible metrics. In the Regge approach, this is provided by varying the link lengths on a fixed lattice. In the dynamical triangulation approach, the link lengths are held fixed but the lattice is varied: all possible triangulations are summed over. Monte Carlo evaluations of the sum are performed by randomly wandering through the space of triangulations. It has been proven that any triangulation can be reached from any other triangulation (for a given topology) through a sequence of local moves [2]. A simple algorithm allows the computation of functional integrals over metrics in any desired dimension with a computer program only 350 lines long [3]. 
The simplest observables in these models are the number of subsimplexes of various dimensions (e.g. nodes, links, triangles, etc.) and these numbers can be used to construct an action. They are related due to topological constraints and the requirement that the triangulations yield a manifold: in three and four dimensions the number of nodes and the number of simplexes determine all of the other numbers of subsimplexes. The basic action used in these theories is

$$
S=\alpha N_{0}-\beta N_{D}
$$

where $\alpha$ and $\beta$ are chemical potentials corresponding to Newton's constant and the cosmological constant, respectively, while $N_{0}$ is the number of nodes and $N_{D}$ is the volume. In two dimensions, $N_{0}$ and $N_{D}$ are related so there is only one term in the action. In fact, it is possible to work in an ensemble where the volume is fixed (leaving zero terms in the action). This is possible because ergodicity has been proven for a restricted set of update moves that do not alter the volume. In three and higher dimensions there is no corresponding proof: the full ensemble including volume fluctuations must be used [4].

To recover a viable continuum theory from the lattice theory requires two limits to be taken. First, there is the thermodynamic, large volume, limit. It is reached by adjusting $\beta$. When $\beta$ is large, the volume is small. As $\beta$ is decreased, the volume increases until a critical value of $\beta$ is reached at which the volume diverges. In practical calculations, $\beta$ must be taken slightly larger than the critical value and there are finite size effects. The other limit that must be taken is the continuum limit. A non-zero correlation length occurs in the continuum, where the lattice spacing is taken to zero, only when the correlation length in lattice units is infinite, so there must be a second-order phase transition present in the phase diagram of the theory. In spin theories and lattice gauge theories, the block spin renormalization group approach is a natural tool with which to study continuous phase transitions [5-11]. This paper develops a renormalization group approach for dynamical triangulations. It is used here to examine the flow of couplings, but it also provides for the systematic calculation of critical couplings and critical exponents if a second-order transition can be found.

As an excellent example of the potential of the renormalization group approach see the application of it to the three-dimensional Ising model in [12]. Before applying this idea to the lattice quantum gravity models, it is useful to consider the differences between the Ising model and a dynamically triangulated model. In the former there is a fixed lattice, of linear dimension $L$, on which the degrees of freedom are arranged in a regular way. It is simple to draw a courser (block) lattice, of linear dimension $L / b$, as a subset of the original one which partitions the spins into identically structured groups ( such as $b$ by $b$ blocks). These groups can then be averaged according to a specified rule in order to convert the original spin configuration $C$, composed of $L^{D}$ spins, into a block spin configuration $C^{\prime}$, composed of $(L / b)^{D}$ spins. In contrast, in a dynamically triangulated model the lattice is not fixed and the lattice structure itself embodies the degrees of freedom. 


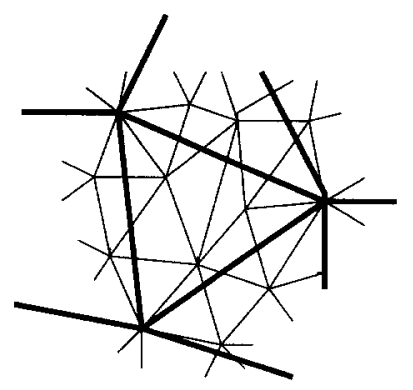

Fig. 1. Bare and block triangulations.

Physically, triangulations are interpreted as embodying information about the metric structure of space-time: points connected by a link are closer than points not connected by a link. Presumably this should be true for block triangulations as well and, in two dimensions, the condition can be imposed easily to define a block spin renormalization group transformation $[13,14]$. This is accomplished as follows. In two dimensions at fixed volume the set of local update moves is a single move, the link flip. View a link and the two triangles that share it as a square with one diagonal drawn. A link flip simply replaces the diagonal with the alternative one. It is the simplicity of the link flipping move that makes it easy to define the block triangulations. Beginning with the original triangulation, $T\left(N_{0}, N_{D}\right)$, illustrated with thin lines in Fig. 1, the $N_{0}^{\prime}$ block nodes are defined as some subset of the original nodes. A block triangulation, $T^{\prime}\left(N_{0}^{\prime}, N_{D}^{\prime}\right)$, illustrated with thick lines in the figure, is required to have the property that the minimum number of links on the original lattice required to travel from one block node to another block node connected to it by a block link must be smaller than the corresponding number of links that must be traversed to travel between the block nodes associated with the alternative diagonal. Block links are flipped until this requirement is satisfied. Once an initial block triangulation has been obtained, updates of the original lattice are followed by updates of the block lattice so as to maintain the rule. The simplest way to obtain an initial block triangulation is to start with a regular original triangulation where it is easy to generate a course lattice with the required property. Matter can also be incorporated into this scheme. This renormalization group transformation gives quite good results for coupling constant flows and critical couplings, but does not do well on critical exponents.

A variety of subsequent renormalization group transformations have been proposed $[15,16]$. The most successful is the node deletion blocking scheme which is very simple and gives excellent results [16]. The idea, still working in two dimensions, is to start with an original triangulation with $N_{0}$ nodes and $N_{2}$ triangles $\left(N_{2}=2\left(N_{0}-\chi\right)\right.$ in two dimensions with $\chi$ the euler characteristic of the surface) and to delete $R$ nodes in order to produce a block triangulation with $N_{0}^{\prime}=N_{0}-R$ nodes and $N_{2}^{\prime}=N_{2}-2 R$ triangles. Consider the deletion of a single particular node labeled $P$. The node $P$ belongs to a number of triangles forming a set to be referred to as $B$. The set $B$ forms a two-dimensional ball around the node $P$. The way $P$ is eliminated is by removing 


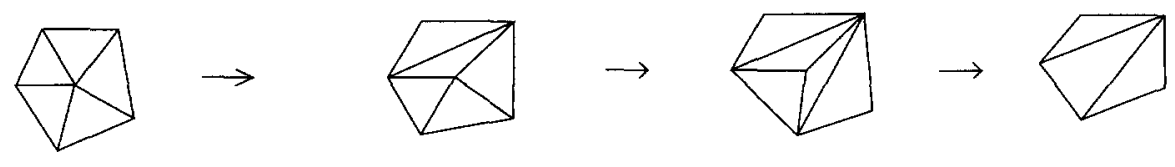

Fig. 2. Node deletion in two dimensions.

the entire ball $B$ from the manifold and then filling the resulting hole back in with new triangles, but without any new nodes. The following algorithm is used to accomplish this. A link attached to $P$ is chosen at random and, if it is an allowed move, flipped. As a result, two old triangles are removed from the set $B$ and one new one is introduced, decreasing the total by one. Links are repeatedly randomly chosen and flipped until there are only three triangles remaining in the ball. Then the node and the remaining three triangles are replaced with one new triangle. The original ball has been completely removed and the hole has been refilled. Fig. 2 schematically represents the procedure. $R$ randomly chosen nodes are deleted in this way and the resulting lattice is interpreted as resulting from a block spin renormalization group transformation. When the standard renormalization group formalism is used to extract critical couplings and exponents for the two-dimensional Ising model coupled to dynamical triangulations, the results agree with the known exact solutions.

Since the node deletion procedure works so well in two dimensions, it is natural to try to apply it in higher dimensions. As mentioned earlier, it is possible to formulate and implement the update moves for triangulations in such a way that the dimensionality of the simplexes is an easily modified variable. The purpose of this paper is to put the node deletion scheme in this more general setting. Obviously, it is simple to identify and eliminate all of the simplexes associated with a given node regardless of the dimension. The tricky part is filling the hole back in. The reason two dimensions at fixed volume is different from a general dimension, $D$, is that the former has only one update move (the link flip) while the latter, using the formulation in [3], has $D+1$. If $i$ ranges from 0 to $D$, then there is a move, for each $i$, that replaces $D+1-i$ simplexes with $i+1$ new ones. $i$ is the dimension of a subsimplex that must be chosen to make the move. For instance, a link flip has $D=2$ and $i=1$ which means a one-dimensional subsimplex (the link) must be chosen to make the move and two old simplexes are replaced with two new ones. In two dimensions, the simplexes associated with a chosen node are eliminated by making link flips which remove simplexes from the node's association until the minimal number are left. The node is then eliminated. This final step is actually an $i=0$ move. In higher dimensions, the set of simplexes associated with a node can be reduced using the $i>0$ moves until the minimal number is reached and a final $i=0$ move completes the operation. By setting up the problem in this way, it is possible to write a program that does node deletion for a simulation of dynamical triangulations in any dimension. Changing dimensions requires the alteration of a single character in the program. 


\section{Details}

This section gives details which can be skipped, but which may be helpful to those who wish to implement the scheme. To produce a block configuration, start by copying the original configuration, along with all relevant bookkeeping, into new data structures. The update routines described in [3] can be modified so that they take arguments specifying which copy of the lattice information is to be changed. In this way, exactly the same routines can be used to update the original lattice and to eliminate nodes in the blocked lattice. Aside from being convenient, this is a good way to avoid errors. In the case of the block lattice, the update moves are not used to generate configurations distributed in accordance with the action but to fill a hole in the manifold made by eliminating a node and its associated simplexes. Create a list of all of the simplexes associated with the node that is to be eliminated. These simplexes form a ball, $B$. Just as in two dimensions where links attached to the node are chosen at random and flipped (when consistent with the geometrical constraints) so as to reduce the number of associated simplexes, in arbitrary dimensions interior $i$-subsimplexes are chosen at random and the update move labeled by $i$ is made. $i$ cannot be zero until the node itself is finally removed.

The interior subsimplex in question is chosen by choosing one of the simplexes in the list at random and then choosing $i+1$ of its nodes at random. The other $D-i$ nodes specify $D-i$ neighboring simplexes because for a marked node in a given simplex there is one other simplex that shares all of the nodes in the given simplex except for the marked one. These neighbors play a role in the algorithm of [3]. Each of the $D-i$ neighboring simplexes has a new node not found in the given simplex and all $D-i$ of these new nodes must be the same if the contemplated move is an allowed one. If the contemplated move passes this test it is referred to as "legal". The $D-i$ neighbors play an additional role here in that they must all be in the list of simplexes associated with the node. If they are not, the chosen subsimplex is not interior to the ball. Altering it would therefore not fill the hole, but would alter its boundary.

Until the last (node eliminating) move, $i$ can be any number from 1 to $D$. In two dimensions at fixed volume, where $i=1$ is the only possibility, there is no choice to be made. In general $D$, many different algorithms for choosing $i$ are possible; this is just the freedom to choose different kernels in the renormalization group transformation. Some may be apt and some may not. Only a single choice for the kernel is explored in this paper. It is intended to eliminate the ball of simplexes as rapidly as possible. Since an $i$ move removes $D-2 i$ simplexes, it is likely that the quickest way to remove the ball is to choose moves with $i$ as small as possible. This is not quite as obvious as it seems because, when a move is made in the interior of the ball, additional simplexes are lost: some of the new ones are no longer associated with the node and therefore do not belong in $B . i=0$ is not an option until the last move, so $i=1$ is probably the best move. However, sometimes no $i=1$ move is allowable, anywhere in the ball, because of pre-existing connections on the surface of the ball due to exterior simplexes. In fact, sometimes the only possible move is node insertion, $i=D$. Consequently, the 
algorithm used here looks for and tries all possible $i=1$ moves, then does the same for all possible $i=2$ moves and so on up to $i=D-1$. If no such moves are possible, a random simplex is chosen from the ball and a node is inserted. After each interior update is made, membership in the ball (defined by having the targeted node as a vertex) is re-examined. Then another interior update is made. This is repeated until the minimal number $(D+1)$ of simplexes are associated with the node which is then removed with an $i=0$ move. (In practice node deletion usually happens quickly. On rare occasions, when simulating in the crumpled phase on large lattices, there are fluctuations to large volumes as the algorithm attempts to remove a node.)

For each move type, all of the simplexes (in random order) are checked for appropriate possibilities. This is done using the fact that a legal move of type $i$ involves $D-i$ neighbors that share the same new node. For a chosen simplex in the ball, all of the new nodes from its neighbors (i.e. those conjugate to nodes in the chosen simplex) that are in the ball are placed in a list which is checked for multiplicity. Each new node with the proper multiplicity corresponds to a possible move. These possible moves are drawn from at random and checked to see if the geometrical constraints are satisfied. If so, the move is made. If not, the list of possible moves is drawn from again. If the list is empty then no move at the given value of $i$ is possible and the procedure must be repeated for the next value of $i$.

Instead of removing a node and its associated simplexes, one might consider removing a link and its associated simplexes or, generally, an $X$-dimensional subsimplex and its associated simplexes. In two dimensions, a node is associated with the curvature. In general, a $(D-2)$-subsimplex is associated with the curvature. Perhaps the appropriate generalization of removing a node in two dimensions is eliminating a $(D-2)$-subsimplex in $D$ dimensions. If the subsimplex chosen for deletion is of dimension $X$ rather than zero, essentially the same procedure as above can be used to fill the hole generated by removing all of the simplexes associated with it. The most important difference is that now only moves of $i>X$ are allowed until the very last move when $i=X$ is allowed. In particular, if $X=D-2$, i.e. subsimplexes associated with the curvature are eliminated, then the lowest allowed value of $i$ is $D-1$ until the last move. $i=D-1$ adds $D-2$ simplexes each move. In two dimensions the volume change is zero which is consistent with the ensemble used there. In higher dimensions the volume increases. One generally expects a renormalization group transformation to produce a smaller system, one with fewer degrees of freedom, so more thought would be necessary to interpret the results in the case of $X=D-2$ with $D>2$. Even $X=1$ empirically appears to produce larger volumes rather than smaller ones in three and four dimensions. Throughout the rest of the paper, $X$ will always be zero.

While deletion of one node is interpreted as a renormalization group transformation, it is generally useful to delete a number of nodes. Perhaps the most naive way to choose the node is to choose a simplex at random and then one of its nodes at random. However, the node chosen in this way is chosen with a probability proportional to its "order", defined to be the number of simplexes that contain it. Choosing different nodes with different probabilities could cause unwanted distortions in the effective theory. One 
remedy is to include a step which only accepts the node with probability equal to the inverse of its order. In this paper, since a large number of deletions are typically used, a list of all of the nodes is constructed and chosen from at random. A brief study suggested that these alternatives have very similar characteristics, both in speed and in results.

Note that two dimensions is special in that the number of nodes and the number of $D$-simplexes (triangles) is related: deleting a node requires the deletion of exactly two triangles. This is useful, because in the block spin renormalization group approach there is typically a small target volume which one wishes to block to. Comparing expectation values only when they are produced on the same size lattice eliminates finite size effects. For instance, if a system of 5000 simplexes is blocked down to 1000 simplexes and the expectation values of that blocked theory are compared to those of a system of 10000 simplexes blocked down to 1000 simplexes, any differences in expectation values of operators are not due to finite size effects (the two systems are the same size) but to differences in the effective theories produced in the two cases. It is easy to see that 2000 nodes must be eliminated in the first case and 4500 in the second.

In higher dimensions, the relationship between the number of nodes and the number of simplexes is dynamically determined. Eliminating all of the simplexes associated with a given node can delete anywhere between $D+1$ simplexes and a number of them comparable to the volume. To target the volume, it would be necessary to perform a sequence of runs, adjusting the number of deleted nodes each time until finally reaching the desired volume. It is more convenient instead to target the node number. For a given coupling, the number of nodes at various volumes is either known from previous work or can be obtained with a single run. Taking the difference between the starting number of nodes and the target number of nodes gives a very good estimate of the number of nodes that must be deleted from the larger lattice to produce the same number as the smaller lattice. A minor adjustment is often still necessary, since node insertion moves are occasionally needed in the process of eliminating the ball of simplexes associated with a deleted node.

Alternatively, instead of deleting a fixed number of nodes, nodes can be deleted until a target node number is reached. The number of deleted nodes then becomes a fluctuating quantity while the final number of blocked nodes is fixed. A very brief study found that these two schemes give identical answers.

Regardless of which scheme is used, node numbers can typically be matched only to an accuracy of 0.5 , since the number of eliminated nodes is an integer while the initial average number of nodes and the target number of nodes are not. This can be patched with an interpolation of data from two runs, or by noting that the node density is nearly constant over such small changes in the node number. Consistent results are obtained in all cases. 


\section{Results}

Node deletion in two dimensions at fixed volume has been studied in [16]. For pure gravity, the string susceptibility exponent and the Hausdorff dimension were calculated. Flow toward a fixed point was verified using operators built from the local curvature and a measure term was added and shown to be irrelevant. For Ising matter, a fixed point was obtained at the (analytically known) critical coupling and a variety of exponents (the string susceptibility exponent, the magnetic exponent, and the thermal exponent) were correctly computed. These results demonstrate that the node deletion renormalization group transformation is quite apt in two dimensions.

In two dimensions, once the infinite volume limit is obtained by adjusting the cosmological constant, the theory is also critical. In higher dimensions an additional parameter (at least) must be tuned. Given the action, Eq. (1), and tuning the cosmological constant to give infinite volume, the only variable left with which to search for a continuous phase transition, and therefore a continuum limit, is $\alpha$. In both three and four dimensions there is a phase transition as a function of $\alpha$ from a phase where the ratio of the number of nodes to the volume goes to zero as the volume increases to a phase where the same ratio approaches a non-zero constant. In three dimensions the phase transition is first order while in four dimensions the phase transition has been thought to be second order although now there is some evidence that it is first order $[17,18]$. The renormalization group procedure detailed in the last section allows us to study the renormalization group flow of these higher-dimensional theories. Two operators are used to study the renormalization group flows: the volume, $N_{D}$, and the logarithm of the order of the nodes, $M$, defined as

$$
M=\sum_{i \in N_{0}} \ln \left(\frac{O_{i}}{D+1}\right)
$$

The latter operator is associated with a measure term in the sense that if a term of the form $\mu M$ is added to the action, it corresponds to a continuum measure of the form

$$
\prod_{x} g^{\mu / 2}
$$

where $g$ is the determinant of the metric [19].

The target number of nodes used here and in the following sections is the number of nodes associated with an unblocked system with 300 simplexes. Smaller systems have the problem that when a large system is blocked down to that size using a fixed number of node deletions the large fluctuations in the block node number occasionally reach zero.

Fig. 3 shows the expectation values of the two operators as a function of the degree of blocking for various couplings. All of the flows begin in the lower left hand corner, where $\left\langle N_{4}\right\rangle=300$. Subsequent points in the plot correspond to systems with initial volumes of $1000,2000,4000$, and 8000 simplexes, all of which have been blocked down to the indicated number of nodes. Lines have been drawn through points sharing 


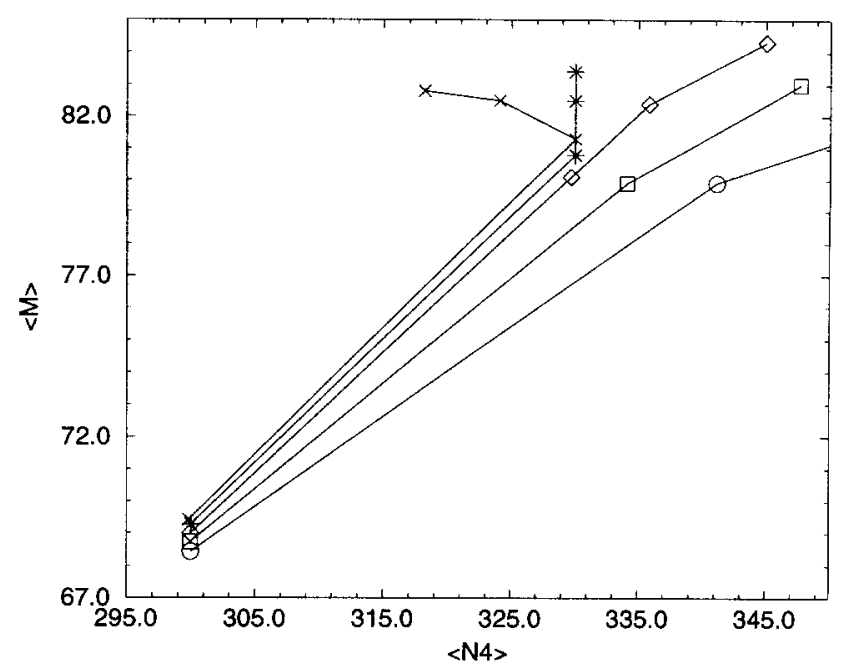

Fig. 3. Renormalization group flows in the four-dimensional dynamical triangulation model near the transition.

a given initial value of $\alpha$. The values of $\alpha$ are (from right to left) 2.3, 2.4, 2.5, 2.6, and 2.7 and are chosen to span the transition region. All runs were for at least a million sweeps each. Errors in the blocked volume, as a function of initial volume, are typically $0.05,0.8,1,3$, and 4 , respectively, with somewhat smaller errors for large $\alpha$ and larger errors for small $\alpha$. The corresponding errors in $\langle M\rangle$ are of order $0.05,0.2,0.4,0.4$, and 0.6 .

Although all of the flows initially head toward the same vicinity, they then split up. For $\alpha=2.3,2.4$ and 2.5, the volume quickly increases with the number of deleted nodes, meaning that the node density quickly flows to zero as the renormalization group transformation is iterated. This is not surprising, since it is the expected large distance behavior in the crumpled phase. For $\alpha=2.7$ the flow turns toward smaller volumes although it is not clear how far it will go: for a given number of nodes, the volume has a lower bound. For $\alpha=2.6$, the volume stays constant for many iterations of the renormalization group, but continues to flow slowly toward increasing $M$. The slow flow near the end of the $\alpha=2.6$ line suggests that a fixed point is nearby and could be reached with a small adjustment in the action. If $\alpha$ is ruled to be insufficient, $\mu$ may be the appropriate parameter to vary. The renormalization group formalism itself can determine the adjustments necessary in $\alpha$ and $\mu$ to obtain a fixed point, but much higher statistics will be necessary to get a useful result (the last two points on the $\alpha=2.6$ line are only 1.5 standard deviations apart).

In four dimensions the peak in the node susceptibility with 8000 simplexes (which moves toward greater $\alpha$ as the volume is increased) occurs around $\alpha=2.3$ [20]. More recent estimates include transition couplings in the range of $2.586(8)$ to $2.654(26)$ depending on assumptions [17]. The results in the figure are compatible with these estimates.

Since the first-order character of the transition is much more firmly established in the 


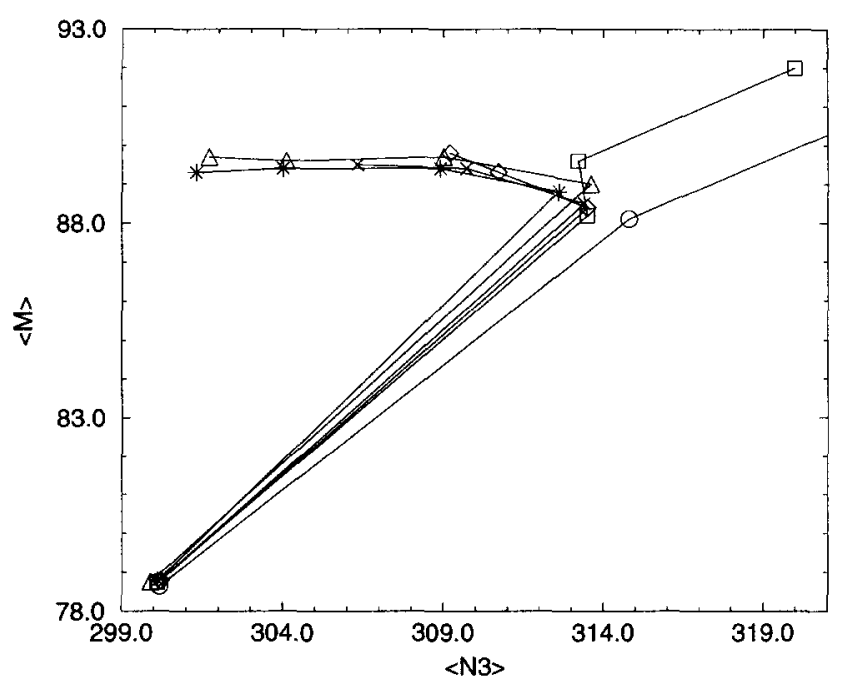

Fig. 4. Renormalization group flows in the three-dimensional dynamical triangulation model near its transition.

case of the three-dimensional dynamical triangulation model, it is interesting to look at the renormalization group flows for this model and to see how they compare to the four-dimensional one. Fig. 4 shows the renormalization group flow lines for the threedimensional dynamical triangulation model for six values of $\alpha: 3.8,3.9,3.95,4.0,4.1$, and 4.2 (labeling the flows from right to left) spanning the transition region. The errors, as a function of initial volume, are typically $0.1,0.4,0.8,1.0$, and 1.2 for the blocked volume and $0.04,0.1,0.4,0.7$, and 0.7 for $\langle M\rangle$. The flows for the first two values of $\alpha$ flow strongly toward large volumes (and therefore small node densities) as is expected in the crumpled phase. Higher values of $\alpha$ flow toward smaller volumes, similar to the smooth phase behavior in four dimensions. There is no intermediate case for the chosen set of initial couplings and therefore no indication of a nearby fixed point. This is consistent with the strongly first-order character of the transition.

Finally, we consider the addition of a measure term to the action. In [19], they suggest that the scaling behavior at the transition changes with the presence of a measure term,

$$
S=\alpha N_{0}-\beta N_{D}+\mu M .
$$

Recent work suggests that the new coupling provides an expanded phase diagram in which there are lines of first-order transitions in both three and four dimensions [21]. A possible phase diagram is given in Fig. 5. The question is: is there a finite value of $\mu^{*}$ ? If so, the endpoint is presumably a second-order phase transition at which a continuum limit can be taken. The nearly second-order nature of the transition in four dimensions suggests that $\mu^{*} \approx 0$ in that case. In three dimensions studies of the specific heat suggest $\mu^{*} \approx-\frac{1}{2}[21]$. As a final illustration of the node deletion scheme, the renormalization group flows are determined for $\mu=-1$. Results are shown in Fig. 6. Typical errors, as a function of the initial volume, are $0.1,0.4,0.6,1$, and 2 for the blocked volume and $0.04,0.1,0.1,0.2$, and 0.2 for $\langle M\rangle$. All of the flows, for several very large couplings 


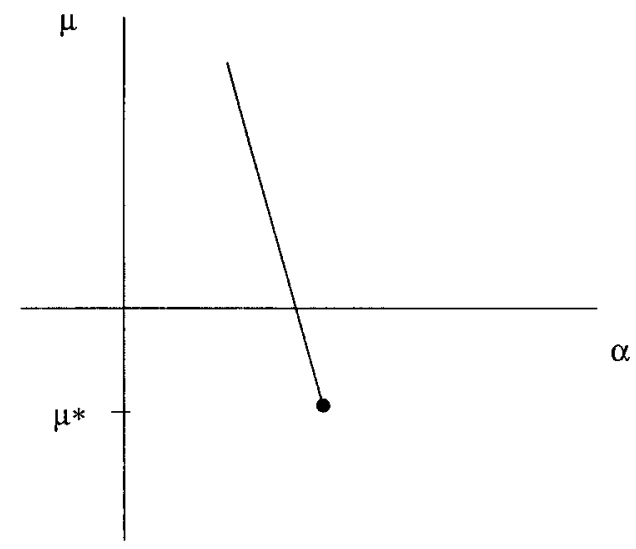

Fig. 5. A possible phase diagram when the action includes a measure term.

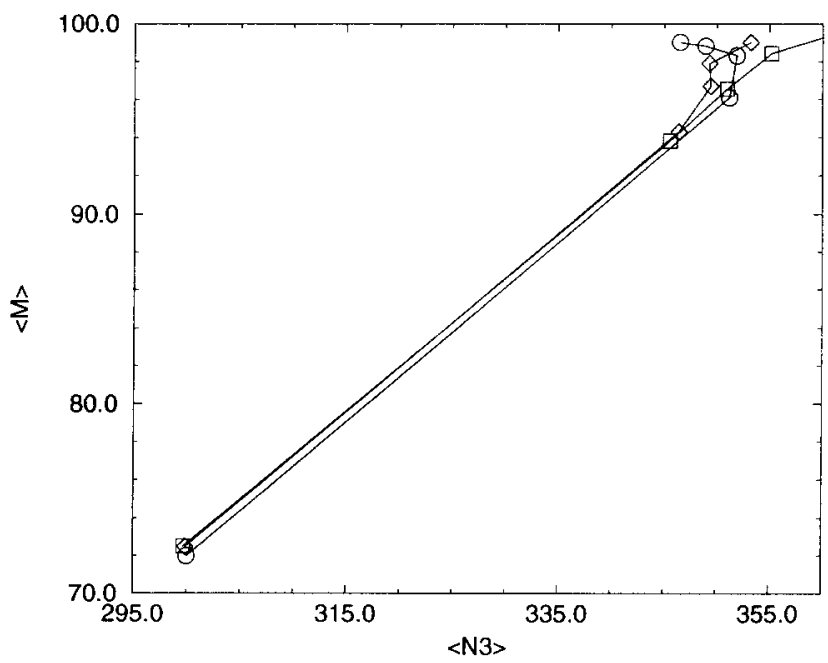

Fig. 6. Renormalization group flows in the three-dimensional dynamical triangulation model with a measure term included.

( $\alpha=5.9,6.4$, and 10.0), where one would expect to find the smooth phase, flow toward small node densities. The largest value of $\alpha$ at the largest blocking level does begin to move to the left. This is not necessarily incompatible with a scenario of flows beyond the end of a first-order line, but it may be that as $\mu$ is made increasingly negative it is necessary to go to larger volumes to see the transition. In this case $\mu^{*}$ would be less than -1 .

If critical endpoints of the first-order lines actually exist, it will take more work to nail down their positions. 


\section{Acknowledgements}

I thank Simon Catterall, John Kogut, and Gudmar Thorleifsson for helpful discussions. This work was supported in part by NSF Grant PHY-9503371.

\section{References}

[1] S. Catterall, Lattice Quantum Gravity: Review and Recent Developments, hep-lat/9510008;

D. Johnston, Gravity and Random Surfaces on the Lattice: A Review, plenary review talk given at Lattice 96: 14th International Symposium on Lattice Field Theory, St. Louis, MO, 4-8 June 1996, Nucl. Phys. B (Proc. Suppl.) 53 (1997), to be published, hep-lat/9607021.

[2] D.V. Boulatov, V.A. Kazakov, I.K. Kostov and A.A. Migdal, Nucl. Phys. B 275 (1986) 641.

[3] S.M. Catterall, Comput. Phys. Comm. 87 (1995) 409.

[4] M. Gross and S. Varsted, Nucl. Phys. B 378 (1992) 367.

[5] K.G. Wilson, Rev. Mod. Phys. 47 (1975) 773.

[6] K.G. Wilson, in Recent Developments in Gauge Theories, ed. G 't Hooft (Plenum Press, New York, 1980).

[7] R.H. Swendsen, Phys. Rev. Lett. 47 (1981) 1775.

[8] R.H. Swendsen, J. Appl. Phys. 53 (1982) 1920.

[9] C. Rebbi and R.H. Swendsen, Phys. Rev. B 21 (1980) 4094.

[10] M.A. Novotny, D.P. Landau and R.H. Swendsen, Phys. Rev. B 26 (1982) 330.

[11] M.E. Fisher, Scaling, Universality and Renormalization Group Theory, in Critical Phenomena Lecture Notes in Physics, Vol. 186, ed F.J.W. Hahne (Springer, Berlin, 1983).

[12] G.S. Pawley, R.H. Swendsen, D.J. Wallace and K.G. Wilson, Phys. Rev. B 29 (1984) 4030.

[13] R.L. Renken, Phys. Rev. D 50 (1994) 5130.

[14] R.L. Renken, S.M. Catterall and J.B. Kogut, Phys. Lett. B 345 (1995) 422.

[15] D.A. Johnston, J.P. Kownacki and A. Krzywicki, Nucl. Phys. B (Proc. Suppl.) 42 (1995) 728.

[16] G. Thorleifsson and S.M. Catterall, SU-4240-619.

[17] P. Bialas, Z. Burda, A. Krzywicki and B. Petersson, Nucl. Phys. B 472 (1996) 293, hep-lat/9601024.

[18] B.V. de Bakker, hep-lat/9603024.

[19] B. Brugmann and E. Marinari, Phys. Rev. Lett. 70 (1993) 1908.

[20] S.M. Catterall, J.B. Kogut and R.L. Renken, Phys. Lett. B 328 (1994) 277.

[21] S. Catterall, J. Kogut and R. Renken, in preparation. 